11. Ohshima, S., et al. 2002. Expression of osteopontin at sites of bone erosion in a murine experimental arthritis model of collagen-induced arthritis: possible involvement of osteopontin in bone destruction in arthritis. Arthritis Rheum. 46:1094-1101
12. Ihara, H., et al. 2001. Parathyroid hormone-induced bone resorption does not occur in the absence of osteopontin. J. Biol. Chem. 276:13065-13071.

13. Yumoto, K., et al. 2002. Osteopontin deficiency protects joints against destruction in anti-type II collagen antibody-induced arthritis in mice. Proc.
Natl. Acad. Sci. U. S. A. 99:4556-4561.

14. Blom, T., Franzen, A., Heinegard, D., and Holmdahl, R. 2003. Technical comments: comment on

"The influence of the proinflammatory cytokine, osteopontin, on autoimmune demyelinating disease." Science. 299:1845a.

\title{
Salicylic acid: an old dog, new tricks, and staphylococcal disease
}

\section{Mathias Herrmann}

Department of Bacteriology and Hygiene, Institute of Medical Microbiology and Hygiene, University of Saarland, Homburg/Saar, Germany

Aspirin has been shown to cause a reduction in the virulence of Staphylococcus aureus-associated endocarditis. A new study (see the related article beginning on page 222) reveals that salicylic acid, the major metabolite of aspirin, acts at the level of transcription to downregulate the production of fibrinogen, fibronectin, and $\alpha$-hemolysin - virulence factors necessary for bacterial replication in host tissues and, now, potential therapeutic targets.

J. Clin. Invest. 112:149-151 (2003). doi:10.1172/JCI200319143.

One hundred and twenty years after its initial description as the pathogen that causes sepsis and abscesses (1), Staphylococcus aureus remains a dangerous organism. Staphylococcal endocarditis is on the rise (2) and still causes significant mortality (3). The methicillin-resistant S. aureus (MRSA) epidemic has entered a new era due to the spread of MRSA into the community (4) and acquisition of new resistance cassettes with the potential for genetic transfer (5). The advent of fully vancomycin-resistant, methicillin-resistant clinical isolates (6) has further weakened the available armamentarium against this pathogen.

\section{Importance of staphylococcal attachment and invasion in endovascular disease}

$S$. aureus is a nonmotile microorganism with a particular propensity to colonize biologic or artificial substrates using a

Address correspondence to: Mathias Herrmann, Department of Bacteriology and Hygiene, Institute of Microbiology and Hygiene, Building 43, Kirrberger Street, 66421 Homburg/Saar, Germany. Phone: 49-6841-162-3900;

Fax: 49-6841-162-3985; E-mail: mathias.herrmann@uniklinik-saarland.de. Conflict of interest: The author has declared that no conflict of interest exists.

Nonstandard abbreviations used: clumping factor A (ClfA); acetylsalicylic acid (ASA); salicylic acid (SAL). battery of pathogenicity factors (7), allowing for specific bacterial attachment. This can be followed by cellular invasion and subsequent tissue degradation. Several lines of evidence clearly indicate that the interaction with host proteins and platelets is instrumental in the development of disease. A plethora of bacterial factors - either wall bound (8) or secreted $(9,10)$ - mediate binding of and attachment to ECM molecules such as fibronectin, fibrinogen, collagen, and vWF. Work with deletion mutants and complemented heterologous hosts has demonstrated the particular role of adhesins that recognize fibronectin (such as fibronectin-binding protein A) and fibrinogen (such as clumping factor A, ClfA), allowing for cellular invasion and production of experimental endocarditis $(11,12)$, and $g f p$ reporter assays from endocarditis models clearly indicate that activation of global regulators that coordinate adhesin and toxin expression, such as agr and sar, occurs in vivo $(13,14)$.

\section{Distinctive effects of acetylsalicylic acid and salicylic acid on platelets and bacteria}

A particular role of platelets in the pathogenesis of staphylococcal endocarditis has been suggested since the early observation by Durack of bacterial interaction with fibrin-platelet matrices at sites of nonbacterial thrombotic endocarditis (15) and the series of reports by Clawson et al. on the interaction of $S$. aureus with purified platelets (16). In the early 1990s, experiments with surface-activated platelets suggested to our group the importance of fibrinogen and $S$. aureus clumping factor in the bacteria-platelet interaction (17). These observations were subsequently confirmed and extended by use of a lowplatelet-binding mutant expressing a mutated ClfA protein (18) that displays diminished virulence in an endocarditis model (19), and by identification of the secreted fibrinogen-binding proteins $\mathrm{Coa}$ and $\mathrm{Efb}$ in phage-display panning assays (20) (Figure 1).

While these observations pointed toward complex but, according to their adhesive function, rather propathogenic events at the bacteria-endocardium interface, the role of platelets had to be reevaluated after the discovery that they function as specialized inflammatory cells (21) in response to secretion of antimicrobial peptides. In fact, paradoxically, hyperexpression of $\alpha$-toxin by $S$. aureus results in diminished virulence in experimental endocarditis, possibly because of the release of platelet microbicidal proteins (22).

The attributed role of platelets in the disease process that results in endovascular infection has prompted a number of researchers to interfere with platelet function for prevention or treatment of endocarditis. Acetylsalicylic acid (ASA, aspirin) has been used in vitro and in a number of experimental models to reduce vegetation sizes and to mitigate the course of disease (23-25). Similar effects have also been observed by Kupferwasser et al. (26). However, when they studied its metabolite, salicylic acid (SAL), in parallel to ASA, they made the interesting observation that pretreatment of bacteria with SAL reduced attachment to the valvular epithelium to an even greater extent than administration of ASA. This observation was accompanied by the in vitro finding that SAL-pretreated $S$. aureus cells bound to 


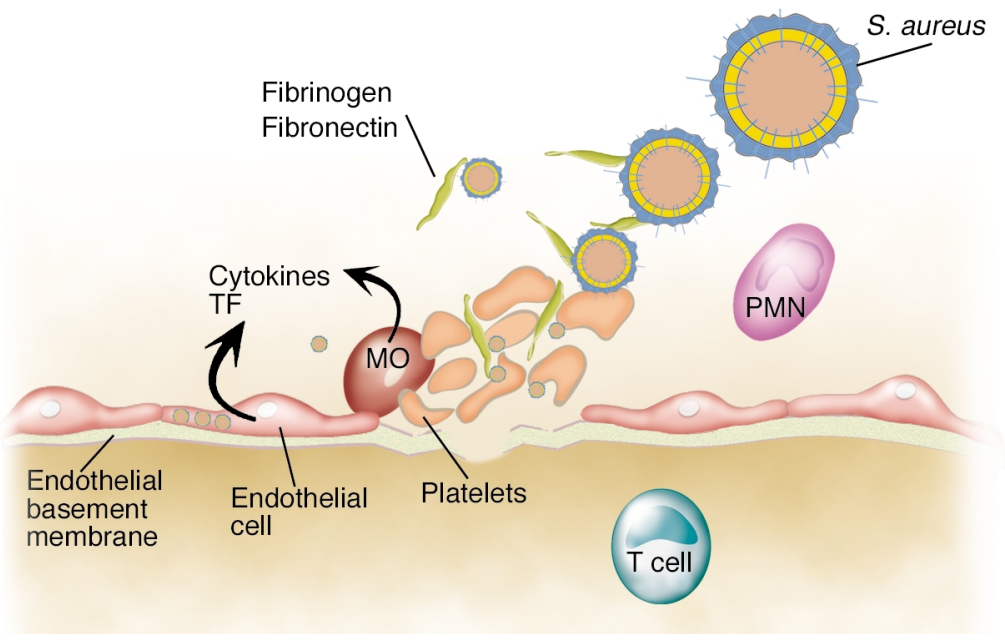

a lesser degree to platelets and to fibrinplatelet and fibrin matrices than did untreated bacteria and elicited platelet aggregation in a prolonged reaction time. Since SAL lacks the key effect of ASA on platelet function consisting of acetylation of the platelet COX-1, the observed in vivo attenuation and in vitro adherence studies delineated distinct effects of ASA and SAL on platelets and microorganisms, respectively.

\section{SAL modulates key regulatory steps in pathogenesis}

In this issue of the JCI, Kupferwasser and colleagues (27) extend these exciting findings. In a careful approach using a number of strains with functional gene regulator deletions and complementations in various genetic backgrounds, they demonstrated an acid stress-independent, SAL-mediated activation of the alternative staphylococcal stress response gene $\operatorname{sig} B$, and consequently a downregulation of the sar $A$ and agr regulons with a concomitant decrease in the expression of bla and fnbA (Figure 2). These effects resulted in decreased bacterial adherence, and reduced toxin-mediated hemolysis and thrombolysis. Most importantly, SAL pretreatment attenuated the course of disease by decreasing the vegetation weight, the vegetation bacterial density, and the renal bacterial density.

The role of sarA and agr in the course of experimental endocarditis has already been studied previously by this group (28). What is novel in this approach is delineation of the effect of SAL on the global regulators, which induces a downregulated status of functional sarA and agr. The nature of agr as a two-component signal transduction-dependent regulator may allow for autoinduction and bacterial interference (29). Another approach to the attenuation of virulence is inhibition of the electron transport (30) that drives microorganisms in a small-colony variant phenotype, as seen in a more chronic-persistent course of disease such as in cystic fibrosis (31). Yet, the establishing

\section{Figure 2}

\section{Figure 1}

Pathogenic events resulting in endovascular disease. Local inflammation activates the binding of fibronectin by ECs through vascular cell adhesion molecules; platelet activation is triggered by cytokines and tissue factor (TF) secreted by monocytes and ECs. Fibronectin also mediates invasion of ECs by S. aureus, allowing for persistence and intracellular growth shielded from host defense. The inflammatory response mediated by $T$ cells and polymorphonuclear neutrophils (PMNs) may be mitigated by the effect of Eap $(39,40)$. MO, monocyte.

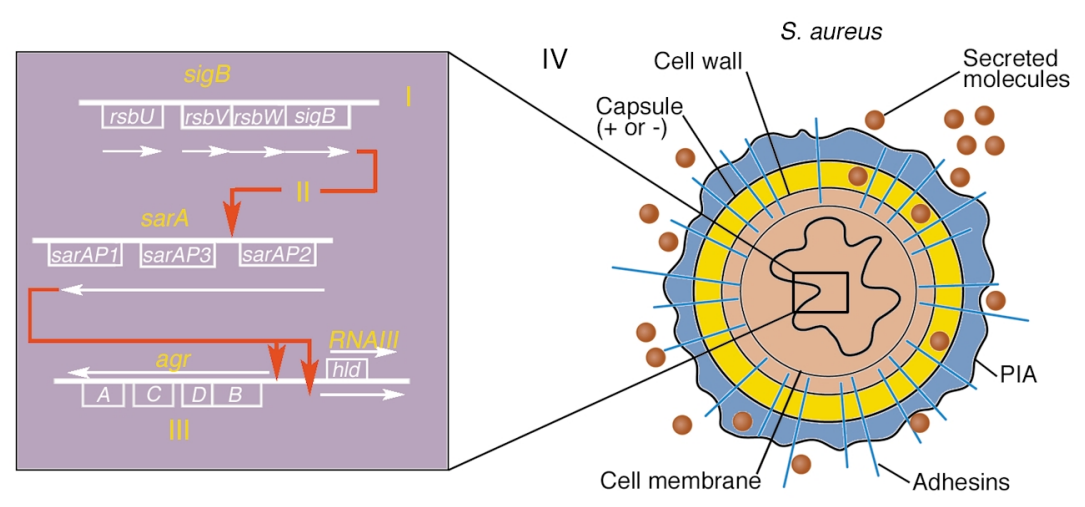

of a straightforward, unequivocal strategy to downregulate staphylococcal virulence using a cheap, simple, relatively nontoxic, resorbable compound such as SAL may be seen as major progress in the development of intervening strategies in addition to antimicrobial drugs.

\section{Potential directions of future research}

The study by Kupferwasser et al. (27) leaves a number of open questions.

Potential roles of salicylic acid (SAL) in the pathogenesis of S. aureus endovascular infection. (I) SAL pretreatment of S. aureus results in overexpression of SigB-dependent genes in both an rsbUpositive and an $r s b U$-negative background, suggesting SigB activation independent of the anti$\sigma$-factor RsbU. (II) Activation of the sarAP3 promotor appears to contribute to decreased expression of active SarA protein, putatively via inhibitory activity of the sarAP3 gene product. Again, SAL appears to contribute to SarA reduction in a sarAP3-additive fashion. (III) As SarA controls expression of RNAll and RNAIII, the net effect of SAL is a mitigation of the agr response. Since $\alpha$-toxin ( $h l a)$ expression depends on both sar and agr, and expression of wall-bound adhesins is also in part controlled by sar, the mitigation of both adhesin and toxin expression in SAL-treated microorganisms appears to depend on a combined sar/agr effect; yet other regulators may also be involved. (IV) In addition to hla, the expression of other secreted molecules such as the extracellular adhesive protein Eap depends on agr and sarA and may be downregulated under the influence of SAL. Expression of the polysaccharide intracellular adhesin (PIA) is suggested to depend on $\operatorname{sig} B$, which may positively control expression of ica $A D B C$, with consequences not yet fully understood in S. aureus endovascular infection. The S. aureus adhesins (FnBPA, FnBPB, ClfA, and $\mathrm{ClFB}$ ) recognize fibronectin and fibrinogen, which are presented on ECs and platelets. 
Firstly, is there any role of SAL in established endocarditis? As downregulation of attachment factors may be of prime importance to prevent initial steps of pathogenesis, SAL may come too late for a salutary effect in treatment. In fact, the above-mentioned "paradoxic" effect due to hyperexpression of $\alpha$-hemolysin may be abrogated, resulting in diminished release of platelet microbicidal proteins. Secondly, how do the findings regarding agr suppression relate to the findings of others, that in a serum milieu (32) or in established infection (33), agr expression is already largely diminished, and other regulators such as sae may play a more prominent role in the in vivo infection? Lastly, what is the mechanism of the effect of SAL on $S$. aureus regulation? SAL is known to exert a plethora of effects on various eukaryotic and prokaryotic cells. More specifically, SAL treatment enhances resistance of S. aureus to fluoroquinolones and fusidic acid. Even more interesting, SAL inhibits biofilm production in Staphylococcus epidermidis (34), apparently because of multiple effects on proteinaceous and nonproteinaceous cell wall and cell surface components (35). Biofilm production in S. aureus (36) and S. epidermidis (37) has been demonstrated to depend on the $i c a A D B C$ gene cluster that confers production of the polysaccharide intercellular adhesin (PIA, also known as PS/A). Expression of the icaADBC gene cluster is environmentally controlled and, at least in part, regulated by sigB. $\operatorname{sig} B$ expression, on the other hand, is controlled by a cascade of sigB activators and inhibitors (38).

The observations by Kupferwasser et al. (27) shed substantially more light onto the patchwork of information concerning the effect of SAL on staphylococci, and they relate it to its potential as a therapeutic compound. Given this exciting new prospect for a widely used and established drug, additional research into the molecular events that result from staphylococcal exposure to SAL is now warranted.

\section{Acknowledgments}

The author's research is supported in part by the Deutsche Forschungsgemeinschaft (DFG Priority Programmes 1047 and 1130) and by a grant from the University of Saarland.
1. Ogston, A. 1882. Micrococcus poisoning. J. Anat 17:24-58.

2. Cabell, C.H., et al. 2002. Changing patient characteristics and the effect on mortality in endocarditis. Arch. Intern. Med. 162:90-94.

3. Bayer, A.S., and Scheld, W.M. 2000. Endocarditis and intravascular infections. In Principles and prac tice of infectious diseases. G.L. Mandell, J.E. Bennett, and R. Dolin, editors. Churchill Livingstone. Philadelphia, Pennsylvania, USA. 857-902.

4. 2003. Outbreaks of community-associated methicillin-resistant Staphylococcus aureus skin infections: Los Angeles County, California, 2002-2003. MMWR Morb. Mortal. Wkly. Rep. 52:88.

5. Daum, R.S., et al. 2002. A novel methicillin-resistance cassette in community-acquired methicillinresistant Staphylococcus aureus isolates of diverse genetic backgrounds. J. Infect. Dis. 186:1344-1347.

6. 2002. Vancomycin-resistant Staphylococcus aureus: Pennsylvania, 2002 [erratum 2002, 51:931] MMWR Morb. Mortal. Wkly. Rep. 51:902.

7. Lowy, F.D. 1998. Medical progress: Staphylococcus aureus infections. N. Engl. J. Med. 339:520-532.

8. Foster, T.J., and Höök, M. 1998. Surface protein adhesins of Staphylococcus aureus. Trends Microbiol. 6:484-488.

9. Palma, M., Haggar, A., and Flock, J.I. 1999. Adherence of Staphylococcus aureus is enhanced by an endogenous secreted protein with broad binding activity. J. Bacteriol. 181:2840-2845.

10. Hussain, M., et al. 2001. Identification and characterization of a novel 38.5-kilodalton secretory protein of Staphylococcus aureus with extendedspectrum binding activitiy for extracellular matrix and plasma. J. Bacteriol. 183:6778-6786.

11. Sinha, B., et al. 2000. Heterologously expressed Staphylococcus aureus fibronectin-binding proteins are sufficient for invasion of host cells. Infect Immun. 68:6871-6878.

12. Que, Y.A., et al. 2001. Reassessing the role of Staphylococcus aureus clumping factor and fibronectin-binding protein by expression in $\mathrm{LaC}$ tococcus lactis. Infect. Immun. 69:6296-6302.

13. Cheung, A.L., Nast, C.C., and Bayer, A.S. 1998 Selective activation of sar promoters with the use of green fluorescent protein transcriptional fusions as the detection system in the rabbit endocarditis model. Infect. Immun. 66:5988-5993.

14. Xiong, Y.-Q., et al. 2002. Activation and transcriptional interaction between agr RNAII and RNAIII in Stapbylococcus aureus in vitro and in an experimental endocarditis model. J. Infect. Dis. 186:668-677.

15. Durack, D.T. 1975. Experimental bacterial endocarditis. IV. Structure and function of the very early lesions. Br. J. Exp. Pathol. 115:81-80.

16. Clawson, C.C, White, J.G., and Herzberg, M.C. 1980. Platelet interaction with bacteria. VI. Contrasting the role of fibrinogen and fibronectin. Am. J. Hematol. 9:43-53.

17. Herrmann, M., Lai, Q.J., Albrecht, R.M., Mosher, D.F., and Proctor, R.A. 1993. Adhesion of Staphy lococcus aureus to surface-bound platelets: role of fibrinogen/fibrin and platelet integrins. J. Infect. Dis. 167:312-322.

18. Siboo, I.R., Cheung, A.L., Bayer, A.S., and Sullam, P.M. 2001. Clumping factor A mediates binding of Staphylococcus aureus to human platelets. Infect. Immun. 69:3120-3127.

19. Sullam, P.M., Bayer, A.S., Foss, W.M., and Cheung, A.L. 1996. Diminished platelet binding in vitro by Staphylococcus aureus is associated with reduced virulence in a rabbit model of infective endocarditis. Infect. Immun. 64:4915-4921.

20. Heilmann, C., Herrmann, M., Kehrel, B.E., and Peters, G. 2002. Platelet-binding domains in 2 fibrinogen-binding proteins of Staphylococcus aureus identified by phage display. J. Infect. Dis. 186:32-39.

21. Yeaman, M.R. 1997. The role of platelets in antimicrobial host defense. Clin. Infect. Dis. 25:951-968
22. Bayer, A.S., et al. 1997. Hyperproduction of alphatoxin by Staphylococcus aureus results in paradoxically reduced virulence in experimental endocarditis: a host defense role for platelet microbicidal proteins. Infect. Immun. 65:4652-4660.

23. Nicolau, D.P., et al. 1993. Reduction of bacterial titers by low-dose aspirin in experimental aortic valve endocarditis. Infect. Immun. 61:1593-1595.

24. Taha, T.H., Durrant, S.S., Mazeika, P.K., Nihoyannopoulos, P., and Oakley, C.M. 1992. Aspirin to prevent growth of vegetations and cerebral emboli in infective endocarditis. J. Intern. Med. 231:543-546.

25. Nicolau, D.P., Tessier, P.R., and Nightingale, C.H 1999. Beneficial effect of combination antiplatelet therapy on the development of experimental Staphylococcus aureus endocarditis. Int. J. Antimicrob. Agents. 11:159-161.

26. Kupferwasser, L.I., et al. 1999. Acetylsalicylic acid reduces vegetation bacterial density, hematogenous bacterial dissemination, and frequency of embolic events in experimental Staphylococcus aureus endocarditis through antiplatelet and antibacterial effects. Circulation. 99:2791-2797.

27. Kupferwasser, L.I., et al. 2003. Salicylic acid attenuates virulence in endovascular infections by targeting global regulatory pathways in Staphylococcus aureus. J. Clin. Invest. 112:222-233. doi:10.1172/ JCI200316876.

28. Cheung, A.L., et al. 1994. Diminished virulence of a sar-/agr-mutant of Staphylococcus aureus in the rabbit model of endocarditis. J. Clin. Invest. 94:1815-1822

29. Novick, R.P. 2003. Autoinduction and signal transduction in the regulation of staphylococcal virulence. Mol. Microbiol. 48:1429-1449.

30. Proctor, R.A., et al. 2002. Two diarylurea electron transport inhibitors reduce Staphylococcus aureus hemolytic activity and protect cultured endothelial cells from lysis. Antimicrob. Agents Chemother. 46:2333-2336.

31. Kahl, B., et al. 1998. Persistent infection with small colony variant strains of Staphylococcus aureus in patients with cystic fibrosis. J. Infect. Dis. 177:1023-1029.

32. Yarwood, J.M., McCormick, J.K., Paustian, M.L., Kapur, V., and Schlievert, P.M. 2002. Repression of the Staphylococcus aureus accessory gene regulator in serum and in vivo. J. Bacteriol. 184:1095-1101.

33. Wolz, C., Goerke, C., Landmann, R., Zimmerli, W. and Fluckiger, U. 2002. Transcription of clumping factor A in attached and unattached Staphylococcus aureus in vitro and during device-related infection. Infect. Immun. 70:2758-2762.

34. Teichberg, S., Farber, B.F., Wolff, A.G., and Roberts, B. 1993. Salicylic acid decreases extracellular biofilm production by Staphylococcus epidermidis: electron microscopic analysis. J. Infect. Dis. 167:1501-1503.

35. Muller, E., Al Attar, J., Wolff, A.G., and Farber, B.F. 1998. Mechanism of salicylate-mediated inhibi tion of biofilm in Staphylococcus epidermidis. J. Infect. Dis. 177:501-503.

36. McKenney, D., et al. 1999. Broadly protective vaccine for Staphylococcus aureus based on an in vivoexpressed antigen. Science. 284:1523-1527.

37. Heilmann, C., et al. 1996. Molecular basis of intercellular adhesion in the biofilm-forming Staphylococcus epidermidis. Mol. Microbiol. 20:1083-1091.

38. Kullik, I., and Giachino, P. 1997. The alternative sigma factor $\sigma^{\mathrm{B}}$ in Staphylococcus aureus: regulation of the $\operatorname{sig} B$ operon in response to growth phase and heat shock. Arch. Microbiol. 167:151-159.

39. Chavakis, T., et al. 2002. Staphylococcus aureus extracellular adherence protein serves as anti-inflammatory factor by inhibiting the recruitment of host leukocytes. Nat. Med. 8:687-693.

40. Lee, L.Y., et al. 2002. The Staphylococcus aureus Map protein is an immunomodulator that interferes with $\mathrm{T}$ cell-mediated responses. J. Clin. Invest. 110:1461-1471. doi:10.1172/JCI200216318. 